\title{
Deoxysphingoid bases as plasma markers in Diabetes mellitus
}

\author{
Mariana Bertea ${ }^{1 \dagger}$, Markus F Rütti ${ }^{3 \dagger}$, Alaa Othman ${ }^{1}$, Jaqueline Marti-Jaun ${ }^{4}$, Martin Hersberger $^{4}$, \\ Arnold von Eckardstein ${ }^{1}$, Thorsten Hornemann ${ }^{1,2^{*}}$
}

\begin{abstract}
Background: Sphingoid bases are formed from the precursors L-serine and palmitoyl-CoA-a reaction which is catalyzed by the serine-palmitoyltransferase (SPT). SPT metabolizes, besides palmitoyl-CoA also other acyl-CoAs but shows also variability towards the use of other amino acid substrates. The enzyme is also able to metabolize alanine, which results in the formation of an atypical deoxy-sphingoid base (DSB). This promiscuous activity is greatly increased in the case of the sensory neuropathy HSAN1, and pathologically elevated DSB levels have been identified as the cause of this disease. Clinically, HSAN1 shows a pronounced similarity to the diabetic sensory neuropathy (DSN), which is the most common chronic complication of diabetes mellitus. Since serine and alanine metabolism is functionally linked to carbohydrate metabolism by their precursors 3-phosphoglycerate and pyruvate, we were interested to see whether the levels of certain sphingoid base metabolites are altered in patients with diabetes.

Results: In a case-control study we compared plasma sphingoid base levels between healthy and diabetic individuals. DSB levels were higher in the diabetic group whereas C16 and C18 sphingoid bases were not significantly different. Plasma serine, but not alanine levels were lower in the diabetic group. A subsequent lipoprotein fractionation showed that the DSBs are primarily present in the LDL and VLDL fraction.

Conclusion: Our results suggest that DSBs are a novel category of plasma biomarkers in diabetes which reflect functional impairments of carbohydrate metabolism. Furthermore, elevated DSB levels as we see them in diabetic patients might also contribute to the progression of the diabetic sensory neuropathy, the most frequent complication of diabetes.
\end{abstract}

\section{Introduction}

Sphingolipids comprise a heterogeneous class of lipids that contribute to plasma membrane and plasma lipoprotein formation. They are derived from the aliphatic amino-alcohol sphingosine, which is commonly formed from the precursors L-serine and palmitoyl-CoA. The condensation of serine with palmitoyl-CoA is a pyridoxalphosphate (PLP) dependent reaction and catalyzed by the enzyme serine palmitoyltransferase (SPT) (EC 2.3.1.50). SPT is a heteromeric enzyme and composed of at least three subunits (SPTLC1, SPTLC2 and SPTLC3) $[1,2]$. The SPTLC2 and SPTLC3 subunits comprise a PLP

\footnotetext{
* Correspondence: thorsten.hornemann@usz.ch

† Contributed equally

${ }^{1}$ Institute for Clinical Chemistry, University Hospital Zurich and Center for Integrative Human Physiology, University of Zurich, Zurich, Switzerland Full list of author information is available at the end of the article
}

consensus sequence which is absent in the SPTLC1 subunit. The product of the SPT reaction, 3-keto-sphinganine, is converted to sphinganine (SA) and subsequently $\mathrm{N}$-conjugated with a second fatty acid to form dihydroceramide (figure 1). The majority of dihydro-ceramide is then desaturated at $\mathrm{C} 4$ to form ceramide, which is the building block for the more complex sphingolipids. Ceramide and to a certain extent also dihydro-ceramide is usually O-linked to a polar head group such as phosphocholine or carbohydrates. This leads to a complex variety of different sphingolipid metabolites. Although L-serine and palmitoyl-CoA are the preferred substrates, the enzyme shows a certain flexibility towards the use of other substrates. Besides palmitoyl-CoA, SPT also metabolizes other acyl-CoAs with a carbon chain length of between $\mathrm{C}_{12}$ and $\mathrm{C}_{18}$. In this, the SPTLC3 subunit shows a higher affinity towards shorter acyl-CoAs (e.g. $\mathrm{C}_{12}$
C Biomed Central

(c) 2010 Bertea et al; licensee BioMed Central Ltd. This is an Open Access article distributed under the terms of the Creative Commons Attribution License (http://creativecommons.org/licenses/by/2.0), which permits unrestricted use, distribution, and reproduction in any medium, provided the original work is properly cited. 


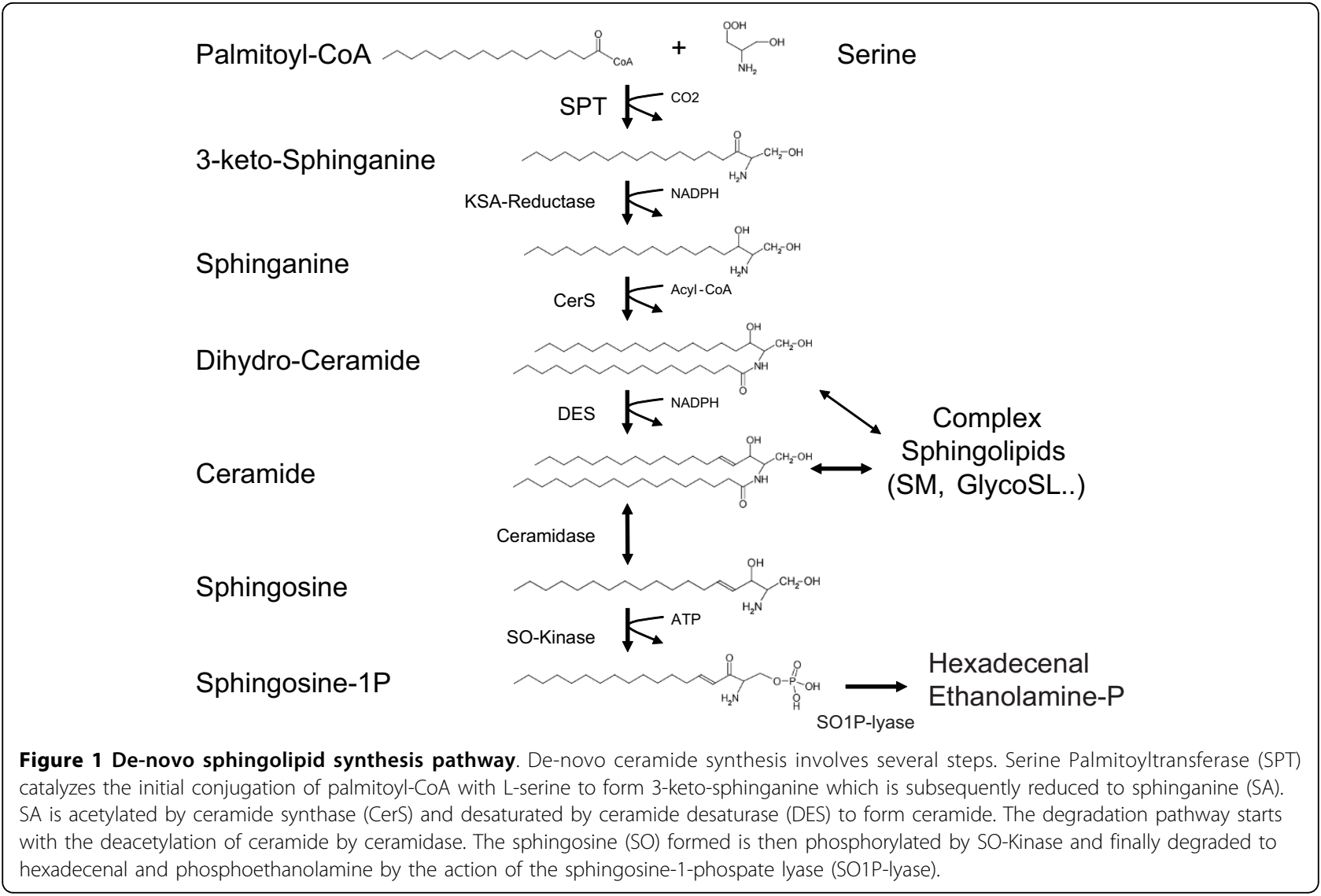

and $\mathrm{C}_{14}$ ) whereas SPTLC2 shows a higher activity with $\mathrm{C}_{16}$ and $\mathrm{C}_{18}$ acyl-CoAs. Both $\mathrm{C}_{18}$ sphingoid and $\mathrm{C}_{16}$ sphingoid bases have been detected in significant amounts in human plasma [3].

Moreover, SPT shows variability towards the use of other amino acid substrates. Besides L-serine, which is the preferred substrate, the enzyme also metabolizes L-alanine and to a certain extent glycine $[4,5]$. This generates an atypical category of sphingoid bases: the 1-deoxy-sphingoid bases (DSBs). The conjugation of alanine forms deoxy-sphinganine (doxSA), whereas the use of glycine results in the formation of deoxymethylsphinganine (doxmethSA). Both metabolites are devoid of the $C_{1}$-hydroxyl group of $\mathrm{SA}$ and are therefore neither metabolized to complex sphingolipids nor degraded by the regular sphingolipid catabolism, since sphingosine-1P as a catabolic intermediate cannot be formed from DSBs.

The activity of SPT with alanine and glycine is greatly increased in the presence of several SPT missense mutations which are associated with the inherited sensory neuropathy HSAN1 (OMIM162400). HSAN1 is an autosomal dominantly inherited axonal neuropathy that is clinically characterized by a loss of pain and temperature sensation, usually starting in the lower limbs and often accompanied by neuropathic pain attacks and skin ulcers. The mutant SPT in HSAN1 shows a highly increased activity with alanine and glycine compared to the wildtype SPT. Consequently, these lipids are found at elevated levels in cells and plasma from HSAN1 patients [4]. Significantly elevated DSB levels were also found in plasma and PNS tissue of the HSAN1 mouse model [6]. HSAN1 mice are transgenic for the mutant SPT and develop a sensory neuropathy within 6-9 months of age. In contrast, double transgenic mice which concomitantly co-express mutant and wildtype SPT are protected and show, in parallel, also significantly lower tissue and plasma DSB levels [6]. The addition of doxSA to DRGs resulted in a dose-dependent reduction of neurite formation in-vitro and in a disruption of the neuronal cytoskeleton structure [4].

Originally, doxSA (also referred to as "Spisulosine" or "ES-285") has been isolated from the arctic clam Spisula polynyma as an investigational marine anticancer drug. It was shown to induce cell death in various breast cancer cell lines $[7,8]$ and to interfere with stress fibre formation and cytoskeleton dynamics by affecting Rho/ Rac-GTPase signaling cascades [9].

Clinically, HSAN1 shows a pronounced similarity to the diabetic sensory neuropathy (DSN), which is the 
most common chronic complication of diabetes mellitus. Both diseases have a late onset and slow progression and typically affect the distal extremities first. The degeneration of small sensory fibers results in the loss of pain sensation, which in turn leads to painless injuries [10].

In this context it is important to note that DSBs are not exclusively formed by the mutant but also by the wildtype SPT - however to a lesser extent [11]. Comparing the kinetics between mutant and wildtype SPT showed that both forms have similar Km's for serine and alanine but that Vmax for alanine is greatly increased in the HSAN1 mutants [12]. Significant DSB levels are therefore also detected in the plasma of healthy individuals [4]. Interestingly, serine and alanine metabolism is physiologically linked to the cellular carbohydrate metabolism. Serine is formed from 3phosphoglycerate and alanine from pyruvate, which are both intermediates of the glycolytic chain. Within this framework of evidence, we were interested to investigate whether sphingoid base levels are altered in patients with an impaired glucose metabolism such as that seen in diabetes mellitus.

\section{Materials and methods Patients}

A total of 70 men and 29 women from Zurich volunteered to participate in the study. Written informed consent was obtained from all participants and the local Ethics Committees approved the study [13]. The case group consisted of 50 consecutive Caucasian patients with diagnosed diabetes mellitus type II. The control group consisted of 49 Caucasians with no history of diabetes recruited from the general population. Clinical chemistry analysis was carried out on a Roche-Hitachi Modular Clinical Chemistry analyzer using commercial tests from Roche Diagnostics (Rotkreuz, Switzerland).

\section{Lipid extraction, hydrolysis and LC-MS analytic}

Plasma sphingolipids were extracted and analyzed by LC-MS as described earlier [4]

\section{Separation of plasma lipoproteins}

Plasma was isolated from diabetic donors after overnight fasting. Three ml plasma was fractionated on a four step density gradient essentially as described [14]. Ultracentrifugation was performed in a Beckman SW-40 swinging bucket rotor for $24 \mathrm{~h}$ at $41000 \mathrm{rpm}$ at $15^{\circ} \mathrm{C}$. Fractions $(1 \mathrm{ml})$ were collected from the top of the centrifuge tube and analyzed for triglyceride; cholesterol and sphingoid bases were analyzed as described above.

\section{Amino acid analysis}

Plasma amino acid concentrations were analyzed according to the method of Frank and Powers [15]

\section{Statisics}

Significance levels were determined with an unpaired student t-test using Sigma Plot v10.0 (SystatSoftware, Inc.). The correlation matrix was generated in SPSS (SPSS, Switzerland). ROC analysis was performed with XLSTAT (Addinsoft, Inc.)

\section{Results}

Sphingolipids in plasma are usually present in a broad variation of subspecies. Sphingoid bases can be found in the saturated (sphinganine, dihydro-sphingosine) and unsaturated (sphingosine) state and are usually $\mathrm{N}$-acetylated with a second fatty acid. Most of the plasma sphingolipids are also conjugated to O-linked head groups, which divert them analytically into various subgroups. However, for this study we were primarily interested in differences between the individual sphingoid base backbones. We therefore simplified the analysis by subjecting the sphingolipids to a sequential acid and base hydrolysis. Acid hydrolysis specifically breaks the $\mathrm{N}$-alkyl chain, whereas alkaline conditions lead to a release of the O-linked head group. The resulting free sphingoid base metabolites were analyzed by LC-MS. The sum of the individual sphingoid bases reflects the total sphingolipid content in the analyzed samples.

In this study we analyzed the $\mathrm{C}_{16^{-}}, \mathrm{C}_{18^{-}}$and deoxysphingolipid levels in a case-control setup comparing 50 patients with documented diabetes mellitus type II (D) and 49 healthy controls $(\mathrm{C})$. The average age of the control group was slightly younger and included more females than the diabetic group. Results are summarised in table 1.

As reported earlier, the most abundant sphingoid bases in plasma were $\mathrm{C}_{18}$-Sphingosine $\left(\mathrm{C}_{18} \mathrm{SO}\right)$ and $\mathrm{C}_{16}$-Sphingosine $\left(\mathrm{C}_{16} \mathrm{SO}\right)$. The concentrations of the dihydro-forms $\mathrm{C}_{18}$-Sphinganine $\left(\mathrm{C}_{18} \mathrm{SA}\right)$ and $\mathrm{C}_{16}$-Sphinganine $\left(\mathrm{C}_{16} \mathrm{SA}\right)$ were much lower and represented about $1-5 \%$ of the respective sphingosine forms. Deoxy-sphingoid bases were generally minor, representing $0.1-0.5 \%$ of the total plasma sphingoid bases. For the DSBs the saturated (doxSA) and unsaturated (doxSO) metabolites were found at similar concentrations. Deoxymethyl-SA or deoxymethyl-SO were not detected.

We found that the average doxSO levels were significantly higher in the diabetic than in the control group. Also doxSA levels were, although less pronounced, higher in the diabetic group (table 1, figure 2A). On the other hand, $C_{16}$ and $C_{18}$ sphingoid base levels were not significantly different between the two groups. This indicates that certain sphingoid bases are specifically altered under diabetic conditions whereas others remain unchanged. Among the other variables total-and LDL-cholesterol was lower in the diabetic groups, which is explained by the general administration of cholesterol 
Table 1 Baseline characteristics and sphingoid base concentrations for the control and diabetic group

\begin{tabular}{llll}
\hline & Control & Diabetes & P-value \\
\hline & $\mathbf{N}=\mathbf{4 9}$ & $\mathbf{N}=\mathbf{5 0}$ & \\
\hline AGE $(y)$ & $57.84 \pm 11.64$ & $63.31 \pm 7.43$ & 0.01 \\
\hline BMI & $\mathbf{2 4 . 8 3} \pm \mathbf{4 . 0 0}$ & $\mathbf{2 8 . 9 7} \pm \mathbf{4 . 5}$ & $<\mathbf{0 . 0 0 0 1}$ \\
\hline Male $(\%)$ & 54 & 84 & \\
\hline CHOL $(\mathbf{m M})$ & $\mathbf{5 . 7 9} \pm \mathbf{1 . 2 6}$ & $\mathbf{4 . 8 2} \pm \mathbf{1 . 1 1}$ & $<\mathbf{0 . 0 0 0 1}$ \\
\hline HDL $(\mathbf{m M})$ & $\mathbf{1 . 6 4} \pm \mathbf{0 . 5}$ & $\mathbf{1 . 3 0} \pm \mathbf{0 . 3 3}$ & $<\mathbf{0 . 0 0 0 1}$ \\
\hline LDL $(\mathbf{m M})$ & $\mathbf{3 . 4 7} \pm \mathbf{0 . 9 7}$ & $\mathbf{2 . 9 0} \pm \mathbf{0 . 9 5}$ & $\mathbf{0 . 0 0 4}$ \\
\hline TG $(\mathrm{mM})$ & $1.48 \pm 0.93$ & $1.44 \pm 0.67$ & 0.77 \\
\hline CRP $(\mathrm{mg} / \mathrm{l})$ & $2.01 \pm 2.18$ & $2.72 \pm 4.14$ & 0.28 \\
\hline SMOKING $(\%)$ & 42 & 77 & \\
\hline STATINS $(\%)$ & 2 & 70 & \\
\hline C16SO $(\mu \mathrm{M})$ & $11.06 \pm 5.40$ & $10.84 \pm 8.71$ & 0.88 \\
\hline C16SA $(\mu \mathrm{M})$ & $0.35 \pm 0.18$ & $0.30 \pm 0.21$ & 0.20 \\
\hline C18SO $(\mu \mathrm{M})$ & $79.44 \pm 28.58$ & $72.93 \pm 38.76$ & 0.34 \\
\hline C18SA $(\mu \mathrm{M})$ & $2.52 \pm 0.91$ & $2.22 \pm 1.14$ & 0.14 \\
\hline doxSO $(\boldsymbol{\mu M})$ & $\mathbf{0 . 1 2} \pm \mathbf{0 . 0 9}$ & $\mathbf{0 . 1 9} \pm \mathbf{0 . 1 5}$ & $\mathbf{0 . 0 0 5}$ \\
\hline doxSA $(\boldsymbol{\mu M})$ & $\mathbf{0 . 0 6 \pm \mathbf { 0 . 0 4 }}$ & $\mathbf{0 . 0 8} \pm \mathbf{0 . 0 5}$ & $\mathbf{0 . 0 8}$ \\
\hline
\end{tabular}

lowering drugs (e.g. statins) in this group. HDL cholesterol was lower in the diabetic groups whereas triglycerides were not different. Age and smoking had no influence.

Since doxSO showed the most significant changes between the groups we calculated the diagnostic values for this variable. A receiver operator curve (ROC) analysis (figure 2B) revealed an area under the curve (AUC) of 0.726 . This converts into a sensitivity of $61 \%$ and a specificity of $89 \%$ at a doxSO threshold of $0.2 \mu \mathrm{M}$. The AUC for the other variables is summarized in figure $2 \mathrm{C}$.

$\mathrm{C}_{16}$ and $\mathrm{C}_{18}$-sphingoid bases are functionally linked to serine metabolism whereas the DSBs are linked to alanine metabolism. We therefore also analyzed the plasma amino acid levels between the two groups. Total amino acid concentration was in general lower in diabetic patients. The most significant changes were seen for glycine, serine and threonine, whereas other amino acids like alanine, valine or histidine were not altered (see table 2).

The correlation matrix (table 3) for the individual variables showed a strong correlation between the $C_{16}$ and $\mathrm{C}_{18}$ sphingoid base metabolites. Also, doxSA and doxSO correlated well with each other but less so with the other sphingoid bases. For doxSO a weak but significant correlation with $\mathrm{C}_{16}$ and $\mathrm{C}_{18} \mathrm{SA}$ was seen, but not with the much more abundant $\mathrm{C}_{16}$ and $\mathrm{C}_{18} \mathrm{SO}$. This indicates that DSB generation is metabolically independent from $\mathrm{C}_{16}$ and $\mathrm{C}_{18}$-sphingoid base formation. In relation to other markers we observed a correlation of the $\mathrm{C}_{16}$ and $\mathrm{C}_{18}$ sphingoid bases with total-as well as HDL-and LDL-cholesterol. DSBs, in contrast, showed a significant correlation with BMI and triglycerides (TG) and to a certain extent with LDL, but not with HDL.

To gain further insight into the physiology of the DSBs we analyzed their distribution into plasma lipoprotein fractions. Lipoproteins were separated by ultracentrifugation, fractionated and the individual fractions analyzed for cholesterol, triglycerides (TG) and sphingoid bases (figure $2 \mathrm{D}$ ). The $\mathrm{C}_{16}$ - and the $\mathrm{C}_{18^{-}}$ sphingolipids were found in the LDL and HDL but not in the VLDL fraction. In contrast, the DSBs were predominantly found in LDL and VLDL but were nearly absent in HDL. This indicates that the plasma DSBs are of hepatic origin since LDL and VLDL but not HDL primarily originate from the liver.

\section{Discussion}

In this study we demonstrate that deoxy-sphingoid bases are elevated in patients with diabetes whereas the other analysed sphingoid base metabolites like $\mathrm{C}_{16} \mathrm{SA}, \mathrm{C}_{16} \mathrm{SO}$, $\mathrm{C}_{18} \mathrm{SA}$ and $\mathrm{C}_{18} \mathrm{SO}$ were not different. This indicates that impairments in glucose metabolism as it is the base of diabetes are reflected in changes of some but not all sphingoid bases. Sphingolipid metabolism represents a metabolic cross point which interconnects lipid (acylCoA) and amino acid (serine and alanine) metabolism. The cellular serine and alanine production is functionally linked to carbohydrate metabolism by their precursors 3 -phosphoglycerate and pyruvate and thereby indirectly also to the carbohydrate metabolism. Therefore, the observed changes indicate a functional interaction between sphingolipid, glucose and fatty acid metabolism which results in an increased production of DSBs in diabetes.

A limitation of this study is the overrepresentation of patients receiving cholesterol lowering drugs in the diabetic group compared to the controls. Thus an impact of statin treatment on the sphingoid base levels cannot be completely excluded. However, diabetic patients with and without statin treatment show no significant differences in the sphingoid base levels. This indicates that a statin treatment does not significantly influence sphingoid base levels. Nevertheless, the influence of statins needs to be addressed in more detail in further studies.

Overall plasma amino acid levels were found to be lower in the diabetic group compared to controls. Among the most significantly lowered amino acids were threonine and serine. Plasma alanine levels, in contrast, were not significantly different between the two groups. It was recently shown that increasing L-alanine levels stimulate DSB formation, whereas the presence of Lserine suppresses DSB formation and increases the generation of $C_{16}$ and $C_{18}$ sphingoid bases [12]. The lower 
A
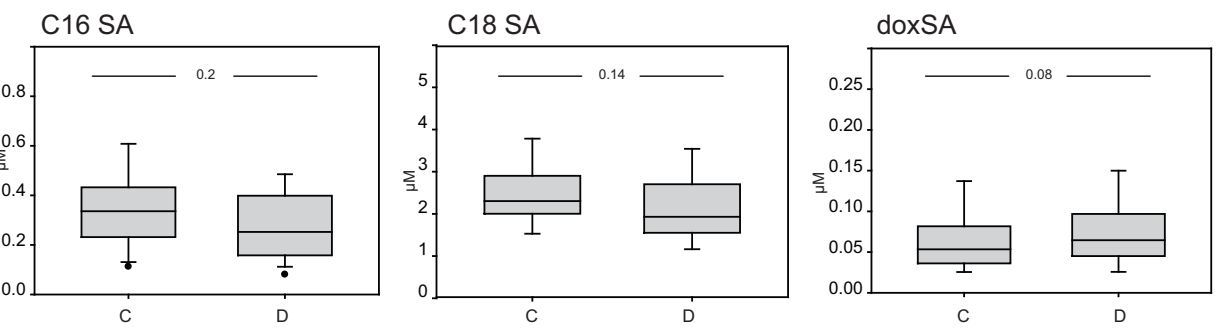

C16 So

\section{C18 So}
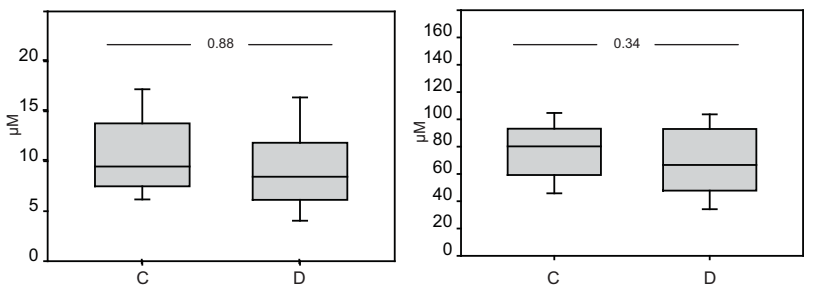

doxSO

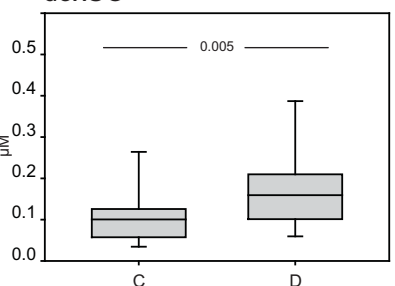

B

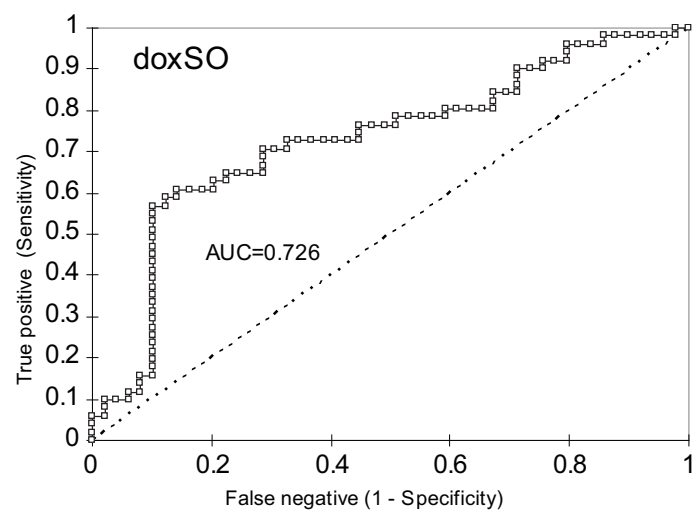

C

\begin{tabular}{|l|c|r|}
\hline & ROC AUC & \multicolumn{1}{c}{ p value } \\
\hline C18 SA & 0.364 & 0.01344 \\
\hline C18SO & 0.394 & 0.05749 \\
\hline doxSA & 0.606 & 0.05894 \\
\hline doxSO & $\mathbf{0 . 7 2 6}$ & $<\mathbf{0 . 0 0 0 1}$ \\
\hline C16 SA & 0.378 & 0.02763 \\
\hline C16SO & 0.431 & 0.22068 \\
\hline age & $\mathbf{0 . 6 9 9}$ & $\mathbf{0 . 0 0 0 2 9}$ \\
\hline CHOL & $\mathbf{0 . 2 9 2}$ & $<\mathbf{0 . 0 0 0 1}$ \\
\hline HDL & $\mathbf{0 . 2 8 0}$ & $<\mathbf{0 . 0 0 0 1}$ \\
\hline LDL & $\mathbf{0 . 3 4 6}$ & $\mathbf{0 . 0 0 3}$ \\
\hline TG & 0.522 & 0.696 \\
\hline
\end{tabular}

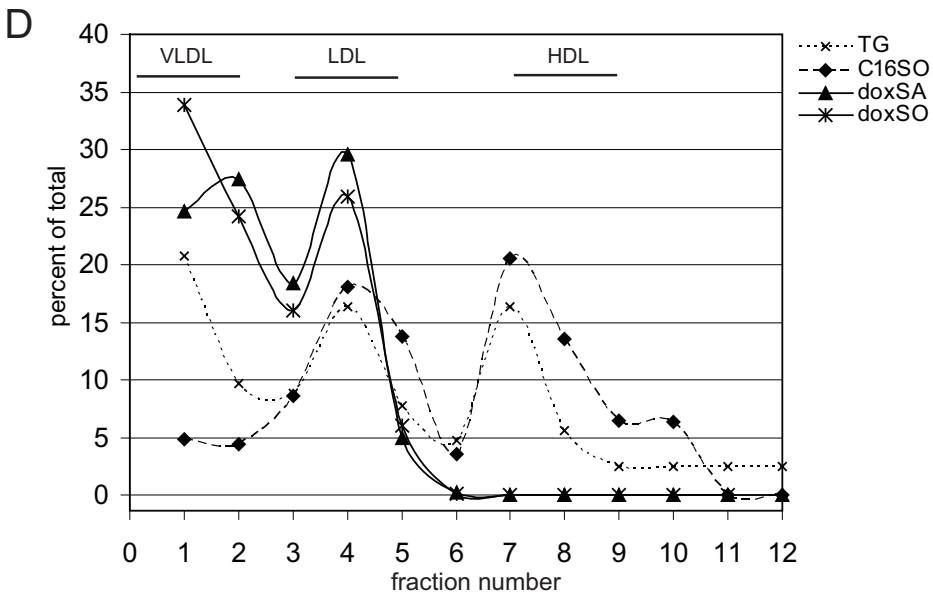

Figure 2 A) Boxplot representation of $C_{16}, C_{18}$ and DSB levels in the control (C) and diabetic (D) cohort. Whereas $C_{16} S A, C_{16} S O, C_{18} S A$ and $\mathrm{C}_{18} \mathrm{SO}$ were not different between the two groups were doxSA and doxSO levels higher in the diabetic group. (Box represents the upper and lower quartile, whiskers show the $5 \%$ and $95 \%$ percentile, the horizontal line represents the median) C) Receiver operator curve (ROC) for doxSO D) Area under the curve (AUC) and p-values for all analyzed variables were calculated from a ROC analysis. Most significant parameters are marked ( $p$ values were calculated with the null hypothesis $(\mathrm{HO})$ as AUC $=0.5$ ). E) Distribution of the DSB and C16 sphingoid bases in the distinct lipoprotein fractions. Human plasma was separated by a four step density gradient ultracentrifugation. The individual fractions were assayed for cholesterol (not shown), triglycerides (TG; dashed line), doxSA (triangle) doxSO (triangle) and $\mathrm{C}_{16} \mathrm{SO}$. Both doxSA and doxSO are only found in the VLDL and LDL but not in the HDL lipoprotein fraction. $\mathrm{C}_{16}-\mathrm{SO}$, in contrast, was present in $\mathrm{LDL}$ and HDL but not in VLDL. The sum of all fractions was defined as $100 \%$ and values are given as a percentage of the total. 
Table 2 Average plasma amino acid concentrations in the control and diabetes group

\begin{tabular}{llll}
\hline & Control & Diabetes & P-value \\
\hline & $\mathbf{N}=\mathbf{4 9}$ & $\mathbf{N}=\mathbf{5 0}$ & \\
\hline Glycine & $\mathbf{4 1 2 . 8 8} \pm \mathbf{1 6 2 . 5 0}$ & $\mathbf{3 0 5 . 4 2} \pm \mathbf{9 2 . 8 0}$ & $\mathbf{0 . 0 0 0 1}$ \\
\hline Alanine & $408.91 \pm 146.71$ & $365.24 \pm 126.73$ & 0.12 \\
\hline Serine & $\mathbf{1 5 1 . 8 3} \pm \mathbf{5 2 . 8 4}$ & $\mathbf{1 2 2 . 7 3} \pm \mathbf{3 4 . 6 4}$ & $\mathbf{0 . 0 0 1}$ \\
\hline Valine & $646.17 \pm 260.35$ & $587.32 \pm 186.66$ & 0.20 \\
\hline Threonine & $\mathbf{1 1 9 . 7 0} \pm \mathbf{4 4 . 4 3}$ & $\mathbf{8 6 . 9 9} \pm \mathbf{2 6 . 5 3}$ & $<\mathbf{0 . 0 0 0 1}$ \\
\hline Isoleucine & $\mathbf{8 5 . 4 5} \pm \mathbf{3 7 . 2 9}$ & $\mathbf{6 9 . 3 0} \pm \mathbf{2 2 . 5 6}$ & $\mathbf{0 . 0 0 9}$ \\
\hline Leucine & $\mathbf{1 5 3 . 5 2} \pm \mathbf{5 8 . 8 3}$ & $\mathbf{1 3 1 . 0 4} \pm \mathbf{4 4 . 7 7}$ & $\mathbf{0 . 0 3}$ \\
\hline Asparagine & $\mathbf{1 1 9 . 1 3} \pm \mathbf{5 8 . 3 0}$ & $\mathbf{8 8 . 4 3} \pm \mathbf{3 3 . 5 8}$ & $\mathbf{0 . 0 0 2}$ \\
\hline Aspartate & $25.84 \pm 12.18$ & $23.38 \pm 11.56$ & 0.30 \\
\hline Glutamine & $\mathbf{3 3 9 . 6 0} \pm \mathbf{2 3 1 . 4 9}$ & $\mathbf{2 5 0 . 5 6} \pm \mathbf{1 6 0 . 3 5}$ & $\mathbf{0 . 0 2}$ \\
\hline Glutamate & $325.21 \pm 160.97$ & $305.92 \pm 120.57$ & 0.50 \\
\hline Methionine & $21.92 \pm 68.40$ & $22.84 \pm 47.48$ & 0.93 \\
\hline Histidine & $\mathbf{1 6 7 . 6 9} \pm \mathbf{7 7 . 6 1}$ & $\mathbf{1 3 4 . 2 0} \pm \mathbf{4 6 . 8 6}$ & $\mathbf{0 . 0 2}$ \\
\hline Arginine & $117.38 \pm 47.40$ & $110.61 \pm 52.52$ & 0.50 \\
\hline Tyrosine & $\mathbf{8 1 . 4 9} \pm \mathbf{3 6 . 5 5}$ & $\mathbf{6 1 . 7 8} \pm \mathbf{2 4 . 8 7}$ & $\mathbf{0 . 0 0 2}$ \\
\hline Tryptophane & $\mathbf{1 0 7 . 2 0 \pm \mathbf { 3 6 . 7 2 }}$ & $\mathbf{8 6 . 9 0} \pm \mathbf{3 2 . 2 6}$ & $\mathbf{0 . 0 0 4}$ \\
\hline
\end{tabular}

Sum

$3283.91 \pm 1137.04 \quad 2752.67 \pm 692.84 \quad 0.0062$

Values $(\mu \mathrm{M})$ are given in mean $+/$ - standard deviation.

serine levels in the diabetic group might therefore explain why DSB levels were higher in the diabetics, since the alanine to serine ratio was decreased in these patients. However, lower levels of serine should then also be associated with lower $\mathrm{C}_{18}$ and $\mathrm{C}_{18}$ sphingoid base levels. This was, by trend, indeed the case although the differences between the two groups were not significant for this cohort.

Earlier reports on amino acid metabolism in obese patients indicate that the situation might be more complex, however. The observation that DSBs are preferentially found in LDL indicates that the plasma DSB levels are primarily of hepatic origin. It has been reported that alanine uptake into the liver is significantly increased in obese individuals [16]. Increased alanine uptake into the liver might thus result in increased DSB formation. However, due to the physiological alanine/glucose shuttle between muscle and liver, the hepatic uptake of alanine is not necessarily reflected in generally lower alanine levels in the venous blood [16].

Besides the direct uptake of alanine into the liver, another mechanism might also contribute to increased hepatic DSB generation. Hepatic glucose uptake is primarily mediated by GLUT2 and is hence insulin independent. Hyperglycemic conditions are therefore associated with high hepatic glucose levels, leading to increased glycolytic flux and production of pyruvate. Hepatic glucose overload might therefore lead to a build-up of pyruvate and its anaerobic conversion into either lactate or alternatively alanine which, in turn, is then converted to doxSA by the hepatic SPT.

This mechanism could also lead to increased DSB generation in other tissues in which glucose uptake is insulin independent, e.g. in kidney, pancreatic beta cells or neurons.

Especially in peripheral neurons elevated DSB levels could contribute to the pathology of diabetic sensory neuropathy (DSN). The inherited neuropathy HSAN1 is caused by a pathological overproduction of DSBs due to several missense mutations in SPT [4]. Interestingly,

Table 3 Correlation matrix of the analyzed variables

\begin{tabular}{|c|c|c|c|c|c|c|c|c|c|c|c|c|c|}
\hline & $\mathrm{C}_{16} \mathrm{SO}$ & $\mathrm{C}_{16} \mathrm{SA}$ & $\mathrm{C}_{18} \mathrm{SO}$ & $\mathrm{C}_{18} \mathrm{SA}$ & doxSO & doxSA & AGE & BMI & CHOL & $\mathrm{HDL}$ & LDL & TG & CRP \\
\hline $\mathrm{C}_{16} \mathrm{SO}$ & & $0.43^{* *}$ & $0.75^{* *}$ & $0.36^{* *}$ & 0.07 & 0.01 & -0.12 & $-0.23^{*}$ & $0.22^{*}$ & $0.27^{* *}$ & 0.18 & -0.09 & -0.10 \\
\hline $\mathrm{C}_{16} \mathrm{SA}$ & $0.43^{* *}$ & & $0.57^{* *}$ & $0.83^{* *}$ & $0.27^{* *}$ & 0.10 & 0.03 & 0.01 & $0.36^{* *}$ & $0.20^{*}$ & $0.35^{* *}$ & 0.13 & -0.10 \\
\hline $\mathrm{C}_{18} \mathrm{SO}$ & $0.75^{* *}$ & $0.57^{* *}$ & & $0.65^{* *}$ & 0.16 & 0.08 & -0.06 & -0.15 & $0.35^{* *}$ & $0.36^{* *}$ & $0.34^{* *}$ & -0.10 & -0.12 \\
\hline $\mathrm{C}_{18} \mathrm{SA}$ & $0.36^{* *}$ & $0.83^{* *}$ & $0.65^{* *}$ & & $0.34^{* *}$ & 0.21 & 0.09 & 0.05 & $0.49^{* *}$ & $0.31^{* *}$ & $0.48^{* *}$ & 0.14 & -0.03 \\
\hline doxSO & 0.07 & $0.27^{* *}$ & 0.16 & $0.34^{* *}$ & & $0.76^{* *}$ & 0.05 & $0.32^{* *}$ & 0.14 & 0.00 & $0.20^{*}$ & $0.29^{* *}$ & 0.11 \\
\hline doxSA & 0.01 & 0.10 & 0.08 & 0.21 & $0.76^{* *}$ & & 0.00 & $0.33^{* *}$ & $0.23^{*}$ & 0.03 & $0.25^{*}$ & $0.27^{* *}$ & 0.18 \\
\hline AGE & -0.12 & 0.03 & -0.06 & 0.09 & 0.05 & 0.00 & & 0.12 & -0.07 & -0.05 & -0.04 & -0.06 & 0.12 \\
\hline BMI & $-0.23^{*}$ & 0.01 & -0.15 & 0.05 & $0.32^{* *}$ & $0.33^{* *}$ & 0.12 & & $-0.27^{*}$ & $-0.45^{* *}$ & -0.11 & 0.04 & $0.41^{* *}$ \\
\hline$\overline{\mathrm{CHOL}}$ & $0.22^{*}$ & $0.36^{* *}$ & $0.35^{* *}$ & $0.49^{* *}$ & 0.14 & $0.23^{*}$ & -0.07 & $-0.27^{*}$ & & $0.49^{* *}$ & $0.92^{* *}$ & $0.44^{* *}$ & -0.03 \\
\hline $\mathrm{HDL}$ & $0.27^{* *}$ & $0.20^{*}$ & $0.36^{* *}$ & $0.31^{* *}$ & 0.00 & 0.03 & -0.05 & $-0.45^{* *}$ & $0.49^{* *}$ & & $0.23^{*}$ & -0.14 & -0.19 \\
\hline$\overline{\mathrm{LDL}}$ & 0.18 & $0.35^{* *}$ & $0.34^{* *}$ & $0.48^{* *}$ & $0.20^{*}$ & $0.25^{*}$ & -0.11 & -0.11 & $0.92^{* *}$ & $0.23^{*}$ & & $0.33^{* *}$ & 0.03 \\
\hline$\overline{\mathrm{TG}}$ & -0.09 & 0.13 & -0.10 & 0.14 & $0.29 * *$ & $0.27^{* *}$ & 0.04 & 0.04 & $0.44^{* *}$ & -0.14 & $0.33^{* *}$ & & 0.08 \\
\hline$\overline{\mathrm{CRP}}$ & -0.10 & -0.10 & -0.12 & -0.03 & 0.11 & 0.18 & 0.12 & $0.41^{* *}$ & -0.03 & -0.19 & 0.03 & 0.08 & \\
\hline
\end{tabular}

The pearson correlations were calculated for the whole dataset combining control and diabetic group. (Correlations $\geq 0.3$ are highlighted bold, ${ }^{*} \mathrm{p}<0.05,{ }^{* *} \mathrm{p}<$ $0.01)$ 
HSAN1 and DSN are clinically very similar: both have a late onset and slow progression and typically affect the distal extremities first. All peripheral nerves are affected, including pain fibers, motor neurons and autonomic nerves. The degeneration of small sensory fibers results in the loss of pain sensation, which in turn leads to painless injuries. Both HSAN1 and DSN are associated with skin ulcers, which is not a common feature in other peripheral neuropathies. The pathological background of DSN is not yet fully understood. Several theories have been discussed and might contribute to unravelling the pathology of DSN. This includes microangiopathy which results in neuronal ischemia, the formation of advanced glycated end products (RAGE) due to a non enzymatic glycosylation of proteins, chronic PKC activation or the increased generation of sorbitol via the polyol pathway, causing oxidative and osmotic stress $[17,18]$. Considering the neurotoxic properties of DSBs, it might therefore be conceivable that the elevated DSB levels in diabetic patients also contribute to the pathology of DSN.

However, in the context of our results, further studies are necessary to validate the role of atypical sphingoid bases as novel biomarkers in diabetes and to address a potential involvement of these metabolites in the pathology of DSN.

\section{Acknowledgements}

The work was undertaken at the University Hospital of Zurich and was supported by grants from the Hartmann Müller Foundation, the Herzog-Egli Foundation, the Olga Mayenfisch Foundation and the Foundation for Scientific Research (University of Zürich) as well as the German Society for Clinical Chemistry and Laboratory Medicine (DGKL), the Gebert Rüf Foundation and the European Commission (LSHM-CT-2006-037631).

\section{Author details}

'Institute for Clinical Chemistry, University Hospital Zurich and Center for Integrative Human Physiology, University of Zurich, Zurich, Switzerland.

${ }^{2}$ Competence Center for Systems Physiology and Metabolic Diseases, Zurich, Switzerland. ${ }^{3}$ Divison of Internal Medicine, Hospital Wil, Wil, Switzerland. ${ }^{4}$ Division of Clinical Chemistry and Biochemistry, University Children's Hospital Zurich and Center for Integrative Human Physiology, University of Zurich, Zurich, Switzerland.

\section{Authors' contributions}

MB performed the lipids analysis; MF performed the amino analysis; AO performed the statistical analysis; JMJ and $\mathrm{MH}$ were involved in study samples collection; AE was involved in the clinical chemistry analytics; $\mathrm{TH}$ participated an the design of the study and wrote the manuscript.

All authors have read and approved the final manuscript

\section{Competing interests}

The authors declare that they have no competing interests.

Received: 9 July 2010 Accepted: 16 August 2010

Published: 16 August 2010

\section{References}

1. Hornemann T, Richard S, Rutti MF, Wei Y, von Eckardstein A: Cloning and initial characterization of a new subunit for mammalian serinepalmitoyltransferase. J Biol Chem 2006, 281(49):37275-37281.
2. Hornemann $T$, Wei $Y$, von Eckardstein $A$ : Is the mammalian serine palmitoyltransferase a high-molecular-mass complex? Biochem J 2007, 405(1):157-164.

3. Hornemann T, Penno A, Rutti MF, Ernst D, Kivrak-Pfiffner F, Rohrer L, von Eckardstein A: The SPTLC3 subunit of serine palmitoyltransferase generates short chain sphingoid bases. J Biol Chem 2009, 284(39):26322-26330

4. Penno A, Reilly MM, Houlden H, Laura M, Rentsch K, Niederkofler V, Stoeckli ET, Nicholson G, Eichler F, Brown RH Jr, et al: Hereditary sensory neuropathy type 1 is caused by the accumulation of two neurotoxic sphingolipids. J Biol Chem 2010, 285(15):11178-11187.

5. Zitomer NC, Mitchell T, Voss KA, Bondy GS, Pruett ST, Garnier-Amblard EC, Liebeskind LS, Park H, Wang E, Sullards MC, et al: Ceramide synthase inhibition by fumonisin B1 causes accumulation of 1-deoxy-sphinganine: A novel category of bioactive 1-deoxy-sphingoid bases and 1-deoxydihydroceramides biosynthesized by mammalian cell lines and animals. J Biol Chem 2009, 284(8):4786-4795.

6. Eichler FS, Hornemann T, McCampbell A, Kuljis D, Penno A, Vardeh D, Tamrazian E, Garofalo K, Lee HJ, Kini L, et al: Overexpression of the wildtype SPT1 subunit lowers Desoxysphingolipid levels and rescues the phenotype of HSAN1. J Neurosci 2009, 29(46):14646-14651.

7. Salcedo M, Cuevas C, Alonso JL, Otero G, Faircloth G, Fernandez-Sousa JM, Avila J, Wandosell F: The marine sphingolipid-derived compound ES 285 triggers an atypical cell death pathway. Apoptosis 2007, 12(2):395-409.

8. Sanchez AM, Malagarie-Cazenave S, Olea N, Vara D, Cuevas C, DiazLaviada I: Spisulosine (ES-285) induces prostate tumor PC-3 and LNCaP cell death by de novo synthesis of ceramide and PKCzeta activation. Eur J Pharmacol 2008, 584(2-3):237-245.

9. Cuadros R, Montejo de Garcini E, Wandosell F, Faircloth G, FernandezSousa JM, Avila J: The marine compound spisulosine, an inhibitor of cell proliferation, promotes the disassembly of actin stress fibers. Cancer Lett 2000, 152(1):23-29.

10. Auer-Grumbach M: Hereditary sensory neuropathy type I. Orphanet J Rare Dis 2008, 3:7.

11. Zitomer NC, Mitchell T, Voss KA, Bondy GS, Pruett ST, Garnier-Amblard EC, Liebeskind LS, Park H, Wang E, Sullards MC, et al: Ceramide synthase inhibition by fumonisin B1 causes accumulation of 1-deoxy-sphinganine: A novel category of bioactive 1-deoxy-sphingoid bases and 1-deoxydihydroceramides biosynthesized by mammalian cell lines and animals. J Biol Chem 2008.

12. Gable K, Gupta SD, Han G, Niranjanakumari S, Harmon JM, Dunn TM: A disease-causing mutation in the active site of serine palmitoyltransferase causes catalytic promiscuity. J Biol Chem 2010, 285(30):22846-22852.

13. Wittwer J, Bayer M, Mosandl A, Muntwyler J, Hersberger M: The C.-292C>T promoter polymorphism increases reticulocyte-type 15-lipoxygenase-1 activity and could be atheroprotective. Clin Chem Lab Med 2007, 45(4):487-492.

14. Kelley $J$, Kruski AW: Density gradient ultracentrifugation of serum lipoproteins in a swinging bucket rotor. Methods Enzymol 1986, 128:170-181.

15. Frank MP, Powers RW: Simple and rapid quantitative high-performance liquid chromatographic analysis of plasma amino acids. J Chromatogr $B$ Analyt Technol Biomed Life Sci 2007, 852(1-2):646-649.

16. Felig P, Wahren J, Hendler R, Brundin T: Splanchnic glucose and amino acid metabolism in obesity. J Clin Invest 1974, 53(2):582-590.

17. Obrosova IG: Update on the pathogenesis of diabetic neuropathy. Curr Diab Rep 2003, 3(6):439-445.

18. Simmons Z, Feldman EL: Update on diabetic neuropathy. Curr Opin Neurol 2002, 15(5):595-603.

\section{doi:10.1186/1476-511X-9-84}

Cite this article as: Bertea et al:: Deoxysphingoid bases as plasma markers in Diabetes mellitus. Lipids in Health and Disease 2010 9:84 